\title{
Genome-wide analysis of the citrus B3 superfamily and their association with somatic embryogenesis
}

\author{
Zheng Liu ${ }^{1,2}$, Xiao-Xia Ge ${ }^{3}$, Xiao-Meng Wu ${ }^{2 *} \mathbb{D}$, Qiang Xu², Ross G. Atkinson ${ }^{4}$ and Wen-Wu Guo ${ }^{2}$
}

\begin{abstract}
Background: In citrus, genetic improvement via biotechnology is hindered by the obstacle of in vitro regeneration via somatic embryogenesis (SE). Although a few B3 transcription factors are reported to regulate embryogenesis, little is known about the B3 superfamily in citrus, and which members might be involved in SE.

Results: Genome-wide sequence analysis identified 72 (CsB3) and 69 (CgB3) putative B3 superfamily members in the genomes of sweet orange (Citrus sinensis, polyembryonic) and pummelo (C. grandis, monoembryonic), respectively. Genome duplication analysis indicated that segmental and tandem duplication events contributed to the expansion of the B3 superfamily in citrus, and that the B3 superfamily evolved under the effect of purifying selection. Phylogenetic relationships were well supported by conserved gene structure and motifs outside the B3 domain, which allowed possible functions to be inferred by comparison with homologous genes from Arabidopsis. Expression analysis identified 23 B3 superfamily members that were expressed during SE in citrus and 17 that may play functional roles at late SE stages. Eight B3 genes were identified that were specific to the genome of polyembryonic sweet orange compared to monoembryonic pummelo. Of these eight B3 genes, CSARF19 was found to be specifically expressed at higher levels in embryogenic callus (EC), implying its possible involvement in EC initiation.
\end{abstract}

Conclusions: This study provides a genome-wide analysis of the citrus B3 superfamily, including its genome organization, evolutionary features and expression profiles, and identifies specific family members that may be associated with SE.

Keywords: Citrus, B3 superfamily, Phylogenetic analysis, Somatic embryogenesis, Callus initiation, Expression profile

\section{Background}

B3 transcription factors (TFs), which contain at least one B3 DNA-binding domain, constitute one of the plantspecific superfamilies $[1,2]$. The B3 domain was initially named according to its position in the third basic region of VIVIPAROUS1 (VP1) from maize [3]. The conserved B3 domain comprises approximately 110 amino acid residues for DNA recognition, consisting of seven $\beta$-barrels and two short $\alpha$-helices $[1,2]$. According to domain structure and

\footnotetext{
* Correspondence: wuxm@mail.hzau.edu.cn

${ }^{2}$ Key Laboratory of Horticultural Plant Biology (Ministry of Education), Huazhong Agricultural University, Wuhan 430070, China

Full list of author information is available at the end of the article
}

phylogenetic analysis, the B3 superfamily is divided into four major families, namely the LAV (LEAFY COTYLEDON2-ABSCISIC ACID INSENSITIVE3-VAL), RAV (RELATED TO ABI3/VP1), ARF (AUXIN RESPONSE FACTOR) and REM (REPRODUCTIVE MERISTEM) families [1]. The B3 superfamily has been characterized in a number of model plants and crops, including Arabidopsis, rice, poplar, Brassica rapa, castor bean, cocoa, soybean, maize, tobacco, grapevine, moss and algae [1, 4-7], but not yet in citrus.

It is reported that B3 TFs from distinct families regulate and control different aspects of plant growth and development. LAV family members, including LEC2 (LEAFY

(c) The Author(s). 2020 Open Access This article is licensed under a Creative Commons Attribution 4.0 International License, which permits use, sharing, adaptation, distribution and reproduction in any medium or format, as long as you give appropriate credit to the original author(s) and the source, provide a link to the Creative Commons licence, and indicate if changes were made. The images or other third party material in this article are included in the article's Creative Commons licence, unless indicated otherwise in a credit line to the material. If material is not included in the article's Creative Commons licence and your intended use is not permitted by statutory regulation or exceeds the permitted use, you will need to obtain permission directly from the copyright holder. To view a copy of this licence, visit http://creativecommons.org/licenses/by/4.0/. The Creative Commons Public Domain Dedication waiver (http://creativecommons.org/publicdomain/zero/1.0/) applies to the data made available in this article, unless otherwise stated in a credit line to the data. 
COTYLEDON2), FUS3 (FUSCA3), ABI3 (ABSCISIC ACID INSENSITIVE3), VAL1 (VP1/ABI3-LIKE 1), VAL2 and $V A L 3$, which each possess a single B3 domain, regulate callus induction, embryo development and phase transition [8-16]. For instance, overexpression of AtLEC2 in transgenic plants induced the formation of callus and somatic embryos [9]. The LAV family generally consists of two subgroups: the LEC2-ABI3 subgroup (LEC2, ABI3 and FUS3) recognizes the $\mathrm{Sph} / \mathrm{RY}$ motif (CATGCA) in the promoters of seed-specific genes $[4,5,17]$, whereas genes in the other subgroup VAL (VAL1, VAL2 and VAL3) which also encode a CW-type zine finger, are expressed in many organs throughout plant development and have central roles in mediating repression of the $L E C 1 / L E C 2-A B I 3$ subgroup network during seed germination $[1,6,11,18]$. RAV family proteins contain a $\mathrm{C}$-terminal $\mathrm{B} 3$ domain that recognizes the consensus sequence CACCTG [19]. Some members of the RAV family also possess an N-terminal AP2/ERF domain that recognizes the consensus sequence CAACA. RAV family members control flowering, organ growth, and have also been shown to be involved in leaf senescence, hormone signaling and responses to various stresses [1926]. The ARF family proteins have an N-terminal B3 domain that recognizes the auxin response element TGTCTC in the promoter of genes responsive to auxin, followed by a highly divergent middle region that determines whether the ARFs act as an activator or repressor [27, 28]. Some ARF proteins contain a conserved carboxyl-terminal interaction domain (Aux/IAA), which is responsible for the dimerization [1]. ARF genes have been widely implicated in auxin-mediated responses during various developmental processes from embryogenesis to flowering, and fruit development [29-35]. REM family members contain at least one copy of the B3 domain, and sometimes up to seven repeats. However, it is not clear whether the B3 domain of the REM protein binds to a specific recognition sequence [36]. The function of REM genes are generally not well understood. Some genes including REM1, VRN1 and VOD have been shown to be involved in floral meristem formation, vernalization and female gametophyte development [37-39].

Citrus is one of the most important fruit crops in the world. However, conventional breeding of citrus is hindered by characteristics such as nucellar polyembryony, long juvenility and male/female sterility [40]. Genetic improvement via biotechnology could be an effective approach, but it is hindered by the barrier of plant regeneration through somatic embryogenesis (SE). Embryogenic callus (EC) can only be induced from the aborted seeds of polyembryonic (apomictic) citrus genotypes, but not from monoembryonic (sexual) genotypes. In addition, the embryonic potential of EC gradually decreases during callus subculture. To understand the mechanisms of $\mathrm{SE}$ and overcome the obstacle of citrus SE, we have conducted a series of studies to identify genes, proteins and
miRNAs involved in citrus SE [41-43]. We found that the B3 domain regulatory network genes CsFUS3, CsABI3 and another B3 gene (CS_P006_E_03) exhibited increased expression during citrus SE induction and formation [41], whereas CsFUS3 was shown to promote citrus $\mathrm{SE}$ by regulating $\mathrm{SE}$-related TFs and hormone pathways, especially ABA and GA pathways [44]. In this study, we performed a genome-wide analysis of the B3 superfamily in polyembryonic sweet orange and monoembryonic pummelo to better understand the regulatory roles of the B3 superfamily genes in citrus SE This comprehensive study of the B3 superfamily should enhance our understanding of possible roles of B3 genes in citrus development, especially in SE.

\section{Results}

\section{Identification and genomic distribution of B3 superfamily} in citrus

A total of $72(C s B 3)$ and $69(C g B 3)$ B3 superfamily TFs were identified in the sweet orange (Citrus sinensis) and pummelo (C. grandis) genomes, respectively (Additional file 1). B3 superfamily members were classified into LAV, RAV, ARF and REM families, then systematically named according to their sequence similarity. In citrus, REM was found to be the biggest B3 family, with 52.8\% (38 CsREMs) and 55.1\% (38 CgREMs) of the total B3 genes identified in sweet orange and pummelo, respectively (Additional file 1). ARFs constituted the second largest family with $26.4 \%$ (19 CsARFs) and 24.6\% (17 CgARFs) of the B3 genes in sweet orange and pummelo. The LAV and RAV families were much smaller, with 11.1\% (8 CsLAVs) and 9.7\% (7 CsRAVs) of B3 genes identified in sweet orange, and 11.6\% (8 CgLAVs) and 8.7\% (6 CgRAVs) of $\mathrm{B} 3$ genes identified in pummelo.

$C s B 3$ TFs were distributed over eight of the nine sweet orange chromosomes. None of the CsB3 genes was located on chromosome 9 (Fig. 1a). The CsB3 gene density per chromosome was variable, with only three genes (4.2\%) (namely CsRAV5, CsARF11 and CsARF17) on chromosome 4, but up to 17 (23.6\%) of the 72 members on chromosome 5. Relatively high densities of CsB3 genes were observed at the chromosome ends, with the highest density at the bottom of chromosome 5 . However, it should be noted, the chromosomal locations for 10 CsB3 genes were not defined because of the incompleteness of sweet orange physical genome map. The distribution and density of $C g B 3$ TFs was also not uniform on the nine chromosomes of pummelo (Fig. 1b). Chromosome 8 contained the largest number of 19 (27.5\%) $C g B 3$ genes, whereas on chromosome 1 there were only three (4.3\%) $C g B 3$ genes.

Orthologous genes of the B3 superfamily between sweet orange and pummelo were not located consistently on the same citrus chromosomes. For example, CsLAV7 was on 


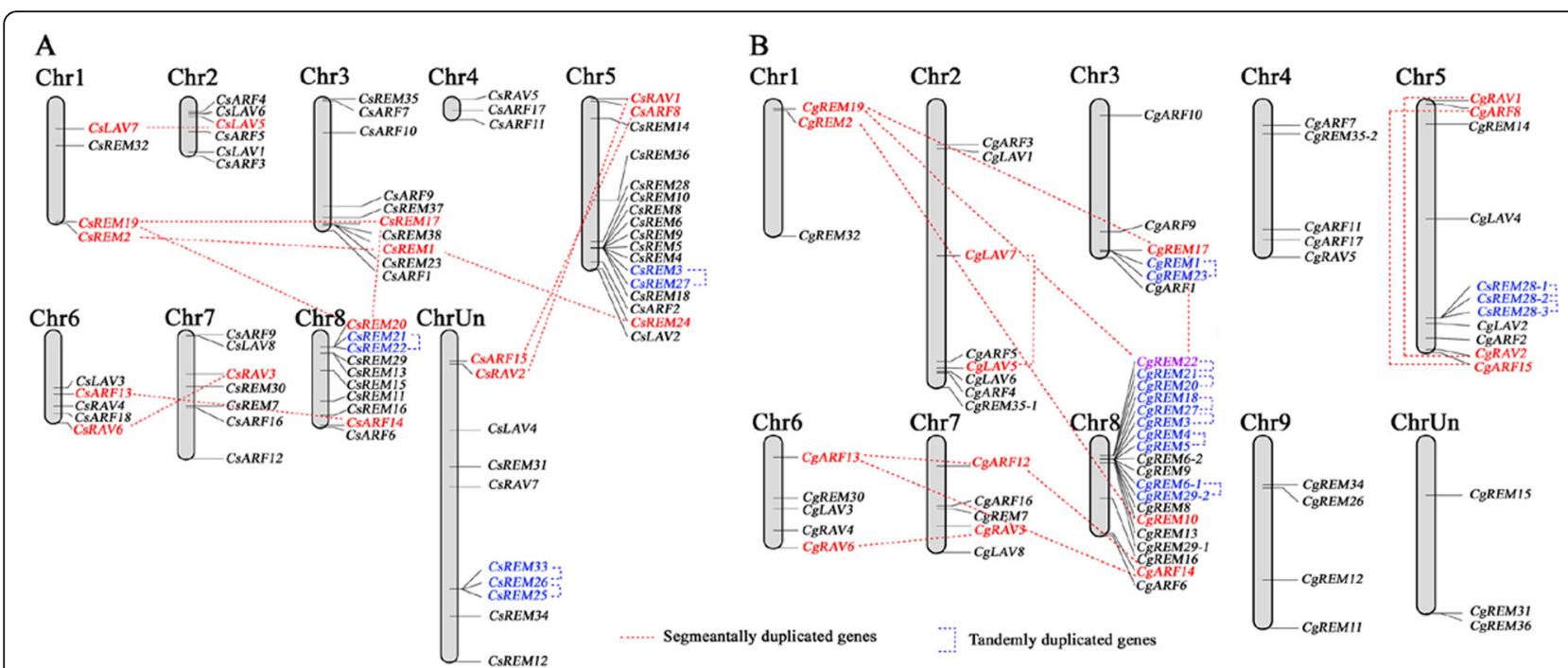

Fig. 1 Chromosomal locations and regional duplication of citrus B3 genes. The chromosomal position of each B3 gene was mapped to the sweet orange (a) and pummelo (b) genomes. The chromosome number is indicated at the top of each chromosome. Segmentally duplicated gene pairs are linked by red dotted lines, whereas tandemly duplicated gene pairs are linked by blue dotted lines

chromosome 1 of sweet orange (Fig. 1a), whereas its orthologous gene CgLAV7 was on chromosome 2 of pummelo (Fig. 1b). These different locations of B3 TFs on chromosomes between citrus species indicated that genetic recombination has occurred extensively in citrus varieties. Among all identified CsB3 genes, a total of ten chromosomal segmental duplication events and four tandem duplication events were identified in the sweet orange genome, whereas in the pummelo genome the corresponding events were 11 and nine respectively (Fig. 1 and Additional file 2), indicating that segmental and tandem duplications may have contributed to the expansion of citrus B3 superfamily. Segmentally duplicated gene pairs (average $\mathrm{Ka} / \mathrm{Ks}=0.22$, where $\mathrm{Ka} / \mathrm{Ks}$ is the nonsynonymous/synonymous substitution ratio) appeared to have undergone extensive intense purifying selection compared to tandemly duplicated gene pairs (average $\mathrm{Ka} / \mathrm{Ks}=$ $0.52)$. The $\mathrm{Ka} / \mathrm{Ks}$ ratios for the majority $(82.4 \%)$ of the duplicated pairs were less than 0.5 , suggesting that the citrus B3 superfamily has evolved under the effect of purifying selection. However, the other two tandemly duplicated gene pairs (CgREM28-1/CgREM28-2 and CgREM6-1/ CgREM29-2) seemed to be under neutral selection, as their $\mathrm{Ka} / \mathrm{Ks}$ ratios were close to 1.0.

To further explore the relationship of B3 superfamily genes between citrus and other plant species, comparative syntenic analyses were conducted in a pairwise manner (Fig. 2), with 37 and 24 collinear B3 gene pairs identified in the sweet orange/Arabidopsis and sweet orange/rice pairs, respectively (Additional file 3). For pummelo/Arabidopsis and pummelo/rice comparisons the corresponding gene pair numbers were 39 and 24 . The number of orthologous events of CsB3/CgB3-AtB3 was higher than that of $C s B 3 / C g B 3-O s B 3$, indicating that the divergence between citrus and Arabidopsis occurred after the divergence of rice and the common ancestor of dicotyledons. It was noteworthy that some B3 collinear gene pairs of citrus/Arabidopsis were anchored to highly conserved syntenic blocks, in which the number of syntenic gene pairs was up to 246, whereas none of syntenic blocks of citrus/Oryza sativa pairs contained more than 20 genes (Additional file 3). The high level of syntenic conservation between the citrus and Arabidopsis indicated that B3 TFs in citrus might share similar structures and functions with orthologs in Arabidopsis.

\section{Characterization of B3 proteins in citrus}

The amino acids length of putative citrus B3 proteins varied widely, ranging from 93 to 1134 (Additional file 1). A few genes had short coding sequence lengths and showed very low expression levels in all samples studied (RPKM $<1$ by RNA-Seq; RPKM: reads per kilobase per million mapped reads) (Figs. 3 and 4), indicating that they may be pseudogenes. The molecular weights and theoretical isoelectric points were also diverse (Additional file 1). The majority of B3 TFs contained only one B3 domain except for some REM family members (Figs. 3d and $4 \mathrm{~d}$ ). A molecular modelling study was then undertaken using the known core structure of the B3 domain crystallized from AtFUS3 (Protein Data Bank code: 6j9b.2; Additional file 4) [45]. Our results showed that the crystal structure had a high degree of sequence identity $(88.46 \%)$ to the experimentally determined template structure, suggesting that a reliable model was generated. The amino acid sequences alignments showed that the B3 domain sequences were highly conserved in 


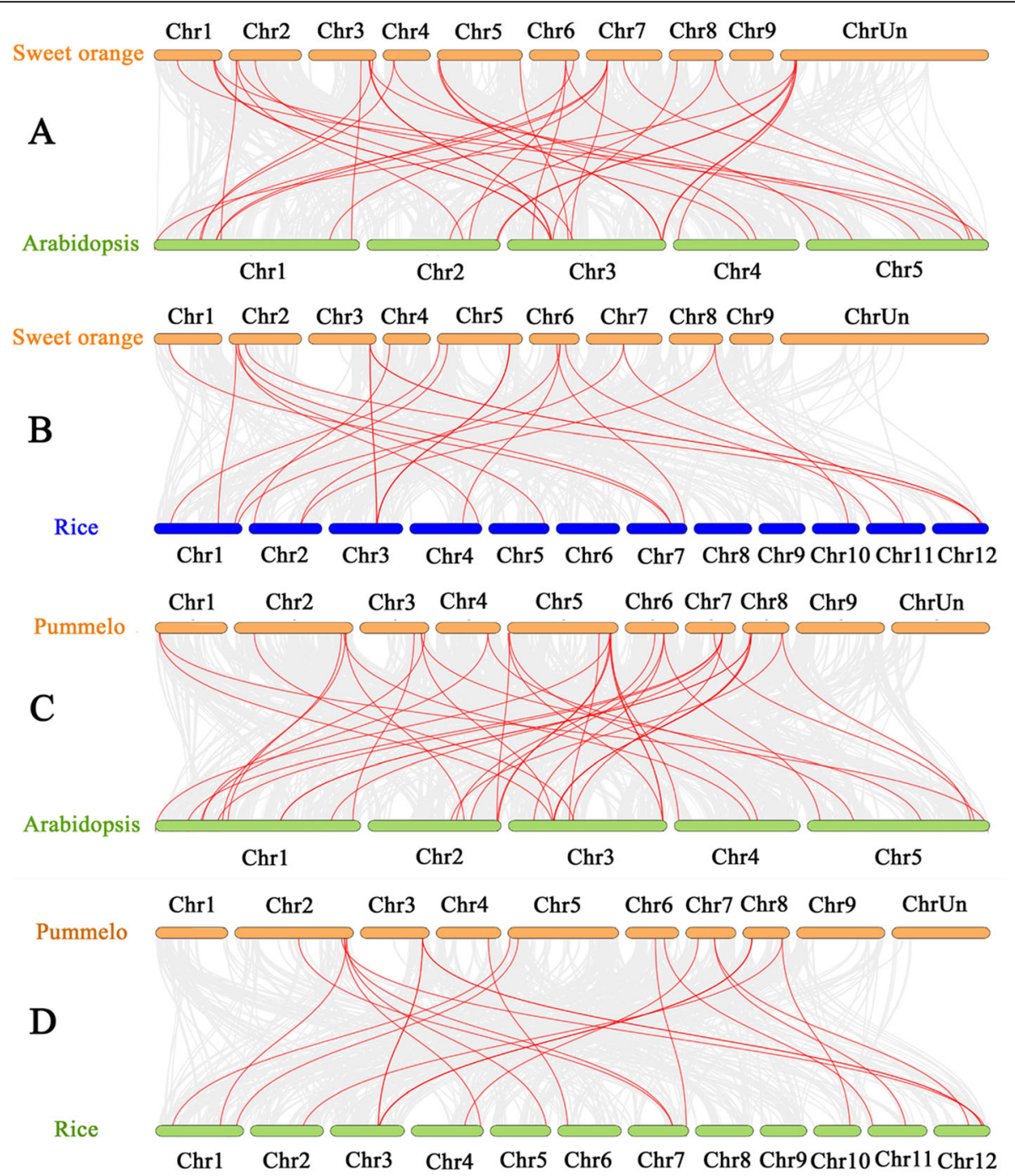

Fig. 2 Gene duplication and synteny analysis of the B3 genes between sweet orange/pummelo and Arabidopsis/rice. Gray lines in the background indicate the collinear blocks within sweet orange/Arabidopsis genomes (a), sweet orange/rice genomes (b), pummelo/Arabidopsis genomes $(\mathbf{c})$, and pummelo/rice genomes $(\mathbf{d})$, respectively. The red lines highlight the syntenic B3 gene pairs

LAV (overall GUIDANCE alignment score $=0.984$ ), RAV (overall GUIDANCE alignment score $=0.906$ ) and ARF families (overall GUIDANCE alignment score $=0.998$ ) (Additional file 5), whereas the B3 domains of REM family exhibited a higher degree of divergence (overall GUIDANCE alignment score $=0.772$ ) (Additional file 6). A total of 20,38, and 24 highly conserved amino acid residues were identical among the B3 domains of all the LAV, RAV, and ARF family members, respectively (Additional file 5). For REM family members, only some conserved amino acid residues including one proline (position $31, \mathrm{P}$ ), two tryptophans (position 72 and 97, W), three glycines (position 70, 96 and 109, G) and three phenylalanines (position 34, 100 and 114, F) were observed in the B3 domains (Additional file 6), which indicated that the B3 domain might have been evolved independently in the REM family.
Phylogenetic analyses of $\mathrm{B} 3$ genes

To explore the phylogenetic relationships of the B3 superfamily, an unrooted phylogenetic tree was constructed among the B3 genes of citrus (sweet orange and pummelo) and the model plant Arabidopsis (Additional file 7). In most subgroups, internal nodes were supported by confidence values of at least $70 \%$, indicative of good consistency in the topology. The tree is in general agreement with Arabidopsis B3 superfamily trees published previously $[1,4]$, which further corroborates the reliability of the tree. In order to test the reliability of the tree topology, protein domain architecture (which was not used in the construction of the tree) were used to provide additional support for the proposed phylogeny. In addition to the B3 domain, other conserved motifs are highly clade specific (Fig. 3d). For example, the ARF and AUX/IAA 


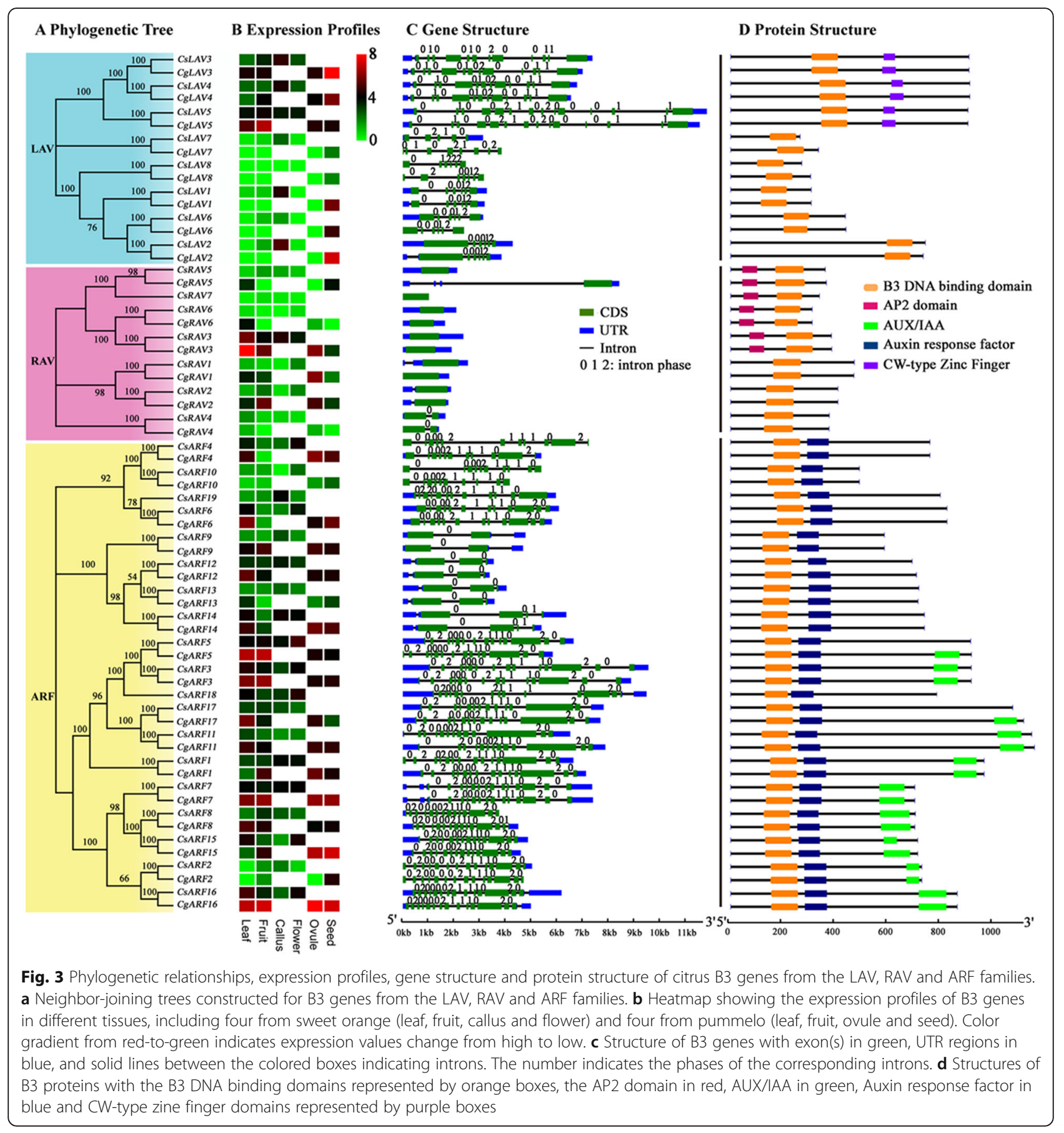

motifs are specifically shared by ARF family. The distribution of the CW-type zinc finger motif supports the tree grouping of CsLAV3/CgLAV3, CsLAV4/CgLAV4 and CsLAV5/CgLAV5 together. Presence of the AP2 domain is also largely clade dependent in the RAV family. The fine structure of the trees is also supported by intron/exon structure data, with a few minor exceptions (Figs. 3c and 4c). For example, all the coding sequences of the ARF genes were disrupted by 2 to 15 introns, while the RAV family contained no more than one intron, except CgRAV5.
According to the classification criteria in Arabidopsis, we divided the members of the major four families into 14 major subgroups (Figs. 3a and 4 a). The LAV family could be subdivided into two subgroups, i.e. LEC2-ABI3 subgroup (I) and VAL subgroup (II). Four CsLAVs in sweet orange (CsLAV1, CsLAV2, CsLAV6 and CsLAV8) and their counterparts in pummelo (CgLAV1, CgLAV2, CgLAV6 and CgLAV8) were clustered with the Arabidopsis LEC2-ABI3 subgroup. The VAL subgroup of four citrus $L A V$ genes (CsLAV3/CgLAV3, CsLAV4/CgLAV4, 


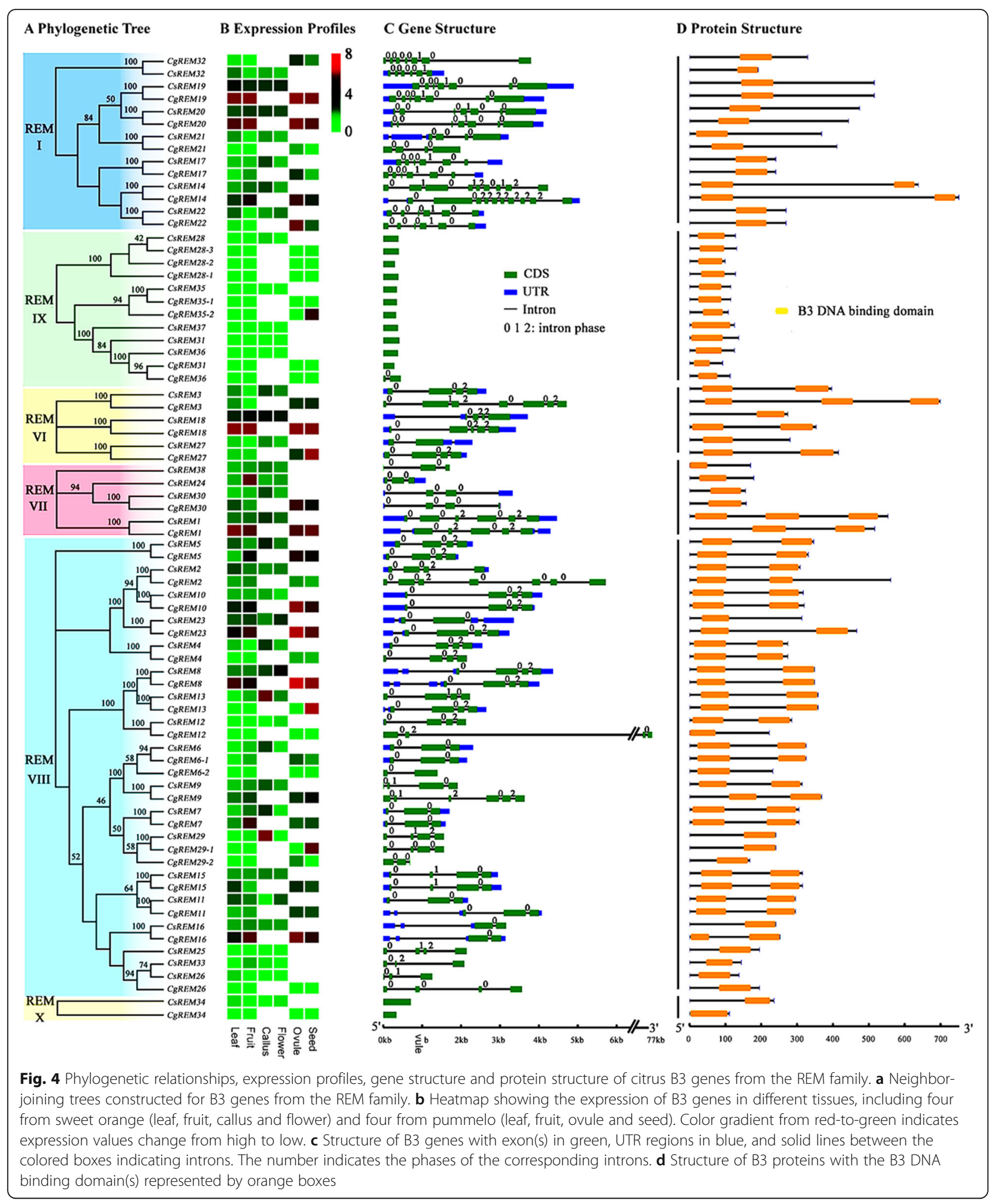

CsLAV5/CgLAV5 and CsLAV7/CgLAV7), which had a conserved B3 domain and a CW-type zinc finger, were clustered with three Arabidopsis VAL proteins (Fig. 3 and Additional file 7).
The RAV family was grouped into two main subgroups based on their phylogenetic relationship. Subgroup I comprised three citrus RAV genes (CsRAVI/ CgRAV1, CsRAV2/CgRAV2 and CsRAV4/CgRAV4) that 
clustered with four AtNGA genes and three AtRAV-like genes from the same branch (Fig. $3 \mathrm{a}$ and Additional file 7). These genes commonly had the conserved B3 domain and contained no more than one intron (Fig. $3 \mathrm{c}$ and d). Subgroup II comprised of four CsRAV genes (CsRAV3, CsRAV5, CsRAV6 and CsRAV7) and three CgRAV genes (CgRAV3, CgRAV5 and CgRAV6), featuring a B3 domain with an upstream AP2 domain (Fig. 4d), and having no introns, except CgRAV5 (Fig. 3c).

Citrus ARF genes were classified into four major subgroups. Subgroup I and II belonged to the same branch, and contained 6 members (CsARF1/CgARF1, CsARF3/ CgARF3, CsARF5/CgARF5, CsARF11/CgARF11, CsARF17/CgARF17 and CsARF18) and 5 members (CsARF2/CgARF2, CsARF7/CgARF7, CsARF8/CgARF8, CsARF15/CgARF15 and CsARF16/CgARF16), respectively (Fig. 3a and Additional file 7). Most of these genes were characterized as having a B3 DNA binding domain, ARF and AUX/IAA domains (Fig. 3d). Subgroup III (CsARF4/CgARF4, CsARF6/CgARF6, CsARF10/CgARF10 and CsARF19) and Subgroup IV (CsARF9/CgARF9, CsARF12/CgARF12-CsARF14/CgARF14) only had the B3 and ARF domains. As most of the REMs in citrus possessed multiple B3 domains and shared low sequence similarity (Fig. 4d and Additional file 6), the phylogenetic analyses were performed within each subgroup of the REM family. The first step of the phylogenetic analysis was comparison of the AtREM sequences with CsREM/ CgREM sequences according to the previous study [4] (Additional file 7). After this initial analysis, six common REM subgroups (REM I and REM VI to REM X) were identified between citrus and Arabidopsis, whereas REM $\mathrm{V}$ (AtREM5) was exclusively identified in Arabidopsis. The vast majority of subgroup I and subgroup II genes contained one B3 domain, and shared homology with the AtREM I and VII type genes, respectively (Fig. 4 and Additional file 7). Subgroup III and IV genes belonged to the AtREM IX and X types, respectively, which possessed only one B3 domain. Subgroup V (AtREM VI) and subgroup VI (AtREM VIII) genes contained several members, the majority of which had more than one B3 domain.

\section{Expression profiles of B3 genes in different tissues and during somatic embryogenesis}

To understand the tissue expression profiles of the B3 genes in citrus, we compared their transcript abundance based on previously published RNA-seq data of different tissues including leaf, fruit, embryogenic callus, flower, ovule and seed from sweet orange and pummelo (Figs. 3b and $4 \mathrm{~b}$ ). Many citrus B3 genes exhibited high transcript abundance level in all five tissues. However, the LEC2ABI3 subgroup and two REM classes (REM IX type and REM X type) exhibited relatively lower expression levels compared with other CsB3 genes. In addition, some of the B3 TFs exhibited tissue-specific expression. For example, CsLAV1/2/6/7, CsARF9/19, CsREM3/4/6/7/9/13/ $14 / 17 / 27 / 28 / 29$ showed the highest transcript abundance in the embryogenic callus (EC), whereas CsREM24 was expressed predominantly in fruit. Some duplicated gene pairs also showed divergent expression profiles. For example, CgARF13 showed a low expression level $(\mathrm{RPKM}=2.76)$ in fruit; whereas its duplicated gene, CgARF14, was highly expressed (RPKM = 56.13) in fruit. These results suggest that duplicated genes may evolve to have diverse functions. Some clustered citrus B3 genes, which were identified as orthologous genes between sweet orange and pummelo species, showed different expression profiles. For example, CgARF17 was mainly expressed in leaf $(R P K M=59.06)$ and ovule (RPKM $=57.40)$ of pummelo, whereas its orthologous gene (CsARF17) in sweet orange showed relatively low expression in all citrus tissues studied, with RPKM values ranging from 4.16 to 7.57 .

To explore the possible involvement of CsB3 genes during citrus SE, the expression profile of 23 CsB3 genes was investigated by qRT-PCR in the six SE stages of 'Valencia' orange, a citrus variety with strong SE capability. These genes were selected based on their relatively high transcript abundance (RPKM values $>10$ ) in EC, or specific accumulation in EC with lower expression level $(1<$ RPKM values $<10)$ according to the RNA-seq data. Based on their expression profiles, these genes could be classified into four types (Fig. 5). The expression of Type I genes was up-regulated during differentiation and showed a highest peak value at the E2 stage (embryogenic callus induced for somatic embryos for 2 weeks; CsARF1, CsARF14, CsREM17 and CsREM18) or E4 stage (embryogenic callus induced for somatic embryos for 4 week; CsLAV1, CsREM4, CsREM5, CsREM13 and CsREM29), and then down-regulated at the early embryo morphogenesis stage (GE, globular embryos), whereas they showed another high peak at the late embryo morphogenesis stage (CE, cotyledon embryos). Type II genes comprise five CsLAVs (CsLAV2, CsLAV3, CsLAV5, CsLAV6 and CsLAV7), one CsRAV (CsRAV3), two CsARFs (CsARF5 and CsARF19) and one CsREM (CsREM27), and were specifically expressed highly at the CE stage, some of which also showed high transcript abundance in one other stage. For Type III genes (CsLAV4, CsARF12 and CsREM6), the mRNA abundance was down-regulated during differentiation stages (E0-E4, embryogenic callus induced for somatic embryos for 0-4 weeks), but was higher at the subsequent stages of embryo morphogenesis (GE or $\mathrm{CE}$ ). Genes in Type IV (CsARF7 and CsREM9) increased progressively throughout the whole SE process.

A total of 15 CsB3 genes which were preferentially expressed in EC were retrieved from the RNA-seq data, including five CsLAVs (CsLAV1 to CsLAV4 and CsLAV7), 


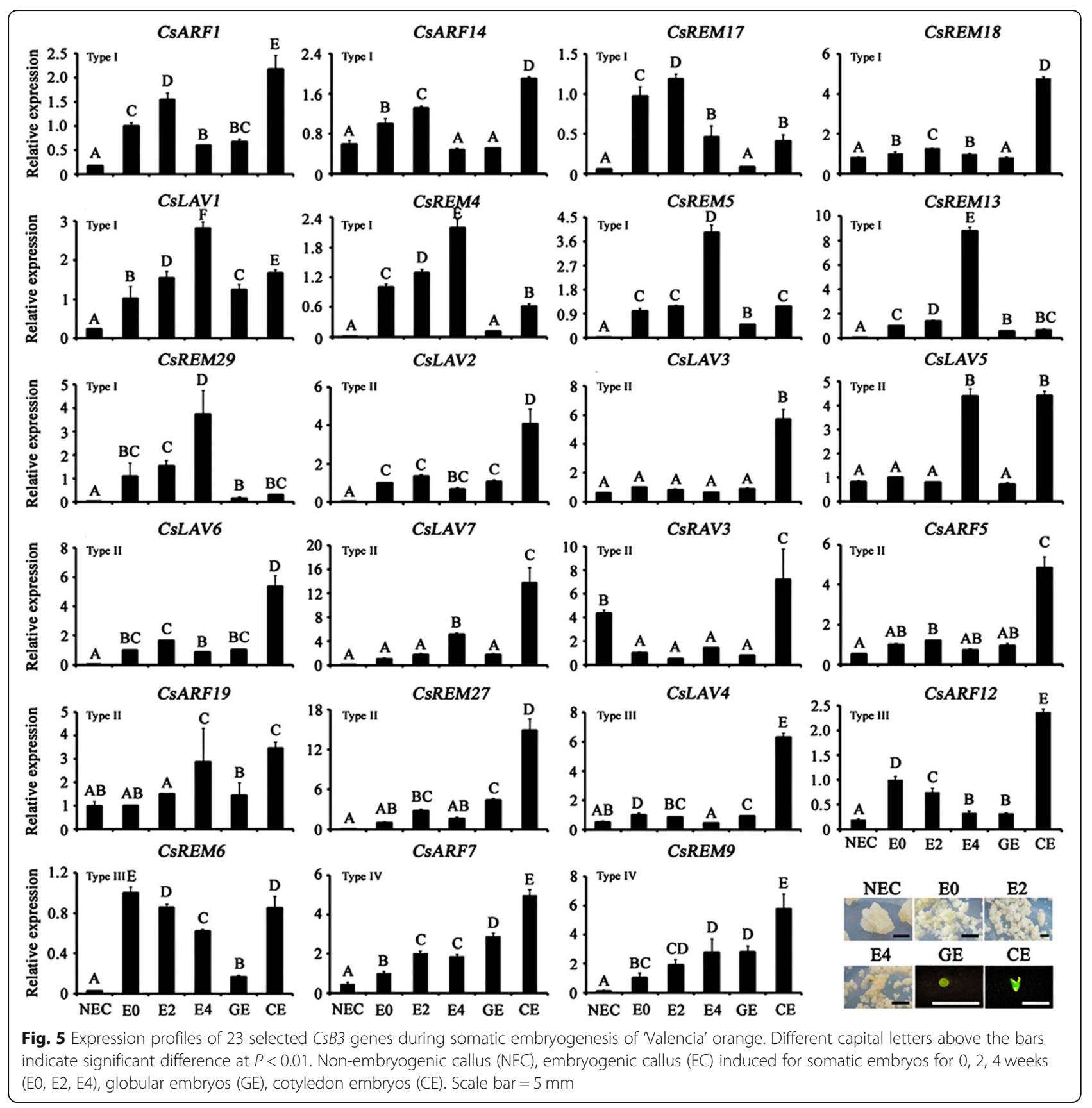

two CsARFs (CsARF12 and CsARF19) and eight CsREMs (CsREM4 to CsREM7, CsREM9, CsREM13, CsREM27, CsREM29) (Fig. 3b, Figs. $4 \mathrm{~b}$ and 6). Among their orthologous genes, eight (five CgLAVs, CgREM13, CgREM27 and CoREM29-1) were preferentially expressed in the ovules and/or seeds of pummelo (Fig. 6), suggesting that these genes may be associated with embryogenesis in vivo and in vitro. Meanwhile, eight B3 genes were identified in the genome of sweet orange, but not in that of pummelo, including CsRAV7, CsARF18, CsARF19, CsREM24, CsREM25, CsREM33, CsREM37 and CsREM38 (Fig. 6). Among them, CsARF19 (Cs7g02210) showed markedly higher expression levels ( $\geq 6$-fold) in EC compared with the other tissues (Fig. 3b), indicating its potential association with callus initiation, because empirically, EC can only be induced from the seeds of the polyembryonic citrus genotypes. With the availability of the citrus genome sequences [46-50], two orthologs of CsARF19, MSYJ162170.1 (amino acids sequence identity of 99.36\%) and Ciclev10030751m (amino acids sequence identity of 99.87\%), were identified in Mangshan mandarin (C. reticulata, a wild mandarin) and Clementine mandarin $(C$. clementina, which is believed to be a chance hybrid of mandarin and sweet orange) $[48,50,51]$, respectively, but 


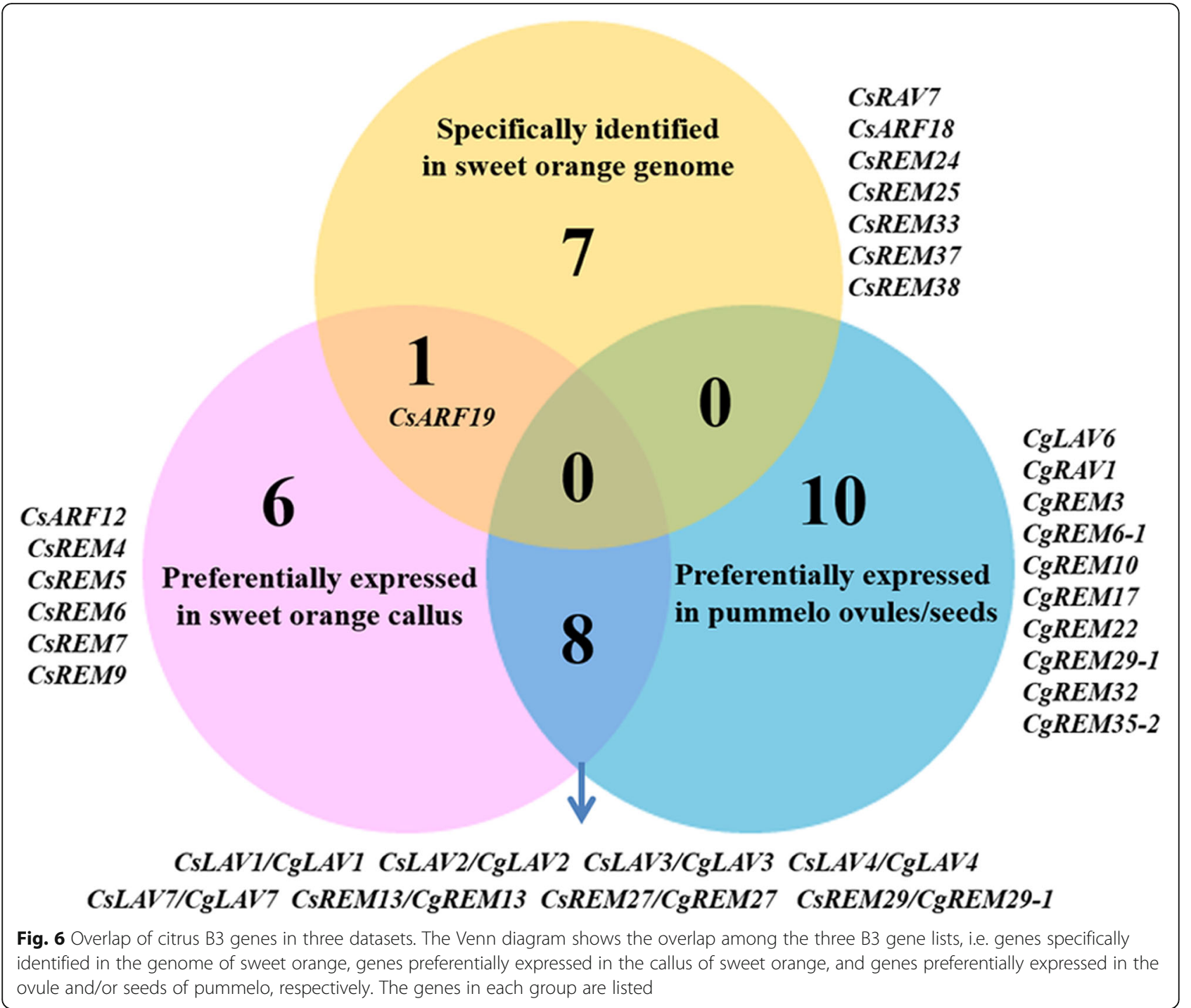

not in Atalantia (Atalantia buxifolia, a primitive citrus), Ichang papeda (C. ichangensis, a wild citrus) and three genera related to citrus, viz. Hongkong kumquat (Fortunella hindsii), trifoliate orange (Poncitrus trifoliata) and citron (C. medica).

\section{Discussion}

The B3 superfamily is one of the largest and most diverse gene families in plants [1]. The evolution of the B3 superfamily has a long history, which can be traced back to the single-celled green algaes Chlamydomonas reinhardtii and Ulva linza, which possess a single B3 gene, strongly suggesting that the B3 domain arose before the development of multicellularity in the plant lineage [1]. In this study, we performed a comprehensive search for B3 superfamily genes throughout citrus genomes. A total of $72 C s B 3$ and $69 C g B 3$ genes were identified, accounting for $0.24 \%$ (29,445 predicted genes in sweet orange) and $0.23 \%(30,123$ predicted genes in pummelo) of all predicted protein-coding genes [46, 47], which was lower compared to the result in Arabidopsis thaliana (110 $A t B 3$ genes out of the 25,498 predicted genes, accounting for $0.43 \%$ ) [52], but higher than that in Oryza sativa (87 OsB3 genes out of the 53,398 predicted gene, accounting for 0.16\%) [53] (Additional file 8). These results showed that the B3 TFs unequally expanded with increasing genome size, since the sizes of the sweet orange and pummelo genomes (367 Mb and $380.76 \mathrm{Mb}$, respectively [46, 47]) are about triple of that of Arabidopsis (125 Mb) [52]. However, there were more identified AtB3 genes in Arabidopsis than in citrus (Additional files 1 and 8), which probably was because of the lack of recent whole-genome duplication (WGD) event in citrus, whereas Arabidopsis has experienced two additional rounds of recent WGD events [46]. 


\section{Possible roles of $C s B 3$ genes during $S E$ of citrus}

Phylogenetic analysis showed that most of the B3 superfamily clades contained both citrus and Arabidopsis proteins (Additional file 7), suggesting that possible functions might be conserved between species. Together with expression pattern analysis of $C s B 3$ genes during $\mathrm{SE}$, it may be possible to identify key regulators of this important process in citrus. Genes with a Type I expression pattern were preferentially expressed during initiation of citrus SE (E0-E4) (Fig. 5). E0-E4 stages are critical stages of differentiation when yellow-green proembryos are generated from the white-yellow EC. It has been demonstrated that CsFUS3 gene (Cs2g14320), a type I $L A V$ family member (CsLAV1), can enhance SE competence of the citrus EC partially by regulating SErelated TFs and hormone pathways, especially ABA and GA pathways [44]. In Arabidopsis, an ortholog (i.e., AtARF5) of another Type I gene CsARF1 (Cs3g25860) is known to promote de novo shoot formation from Arabidopsis callus by pathways involving the downstream functions of STM and CRF2 [54]. Thus, the elevated expression of the Type I B3 genes during SE suggested their possible involvement in citrus SE.

After the formation of GE, the embryoids develop into $\mathrm{CE}$, each with two well-developed cotyledons, which denotes the end of embryo morphogenesis. The CEs undergo phase transition to generate a plantlet through a germination-like process. The majority, but not all, of the Type II and Type III CsB3 genes showed progressively decreasing expression or maintained low levels of expression at EC differentiation stages (E0-E4), but had much higher expression in CE (Fig. 5). The well-studied B3 member AtABI3, which is the putative ortholog of the Type II gene CsLAV2 (Cs5g34660) in Arabidopsis, has been demonstrated to regulate abscisic acidresponsive genes in phase transition from late embryo development to germination $[8,55]$, implying that CsLAV2 may also be functional in late embryogenesis.

The AtLEC2 gene is the best recognized regulator of plant SE $[9,56]$. However, in citrus SE, CsLAV6 (Cs2g05780) (the Type II ortholog of $A t L E C 2$ ) showed constitutively low expression during early stages of SE (EO-GE), and accumulated specifically in CE (Fig. 5). Previous studies have shown that CsLAV6 was not present in suppression subtractive hybridization (SSH) libraries of citrus SE tissues [41], and expression of CsLAV6 is not increased in CsFUS3 overexpressed EC lines in which the SE competence was enhanced [44]. Thus, CsLAV6 may not promote SE initiation like its ortholog $A t L E C 2$, but have a function in late SE.

Three Type II B3 genes, namely CsLAV3 (Cs6g10020), CsLAV5 (Cs2g06770) and CsLAV7 (Cs1g06390), clustered together and shared a high level of sequence similarity with three repressors of embryonic pathways in Arabidopsis (i.e., AtVAL1, AtVAL2 and AtVAL3)
(Additional file 7) [11]. AtVAL1-3 proteins are required for repression of LAFL TFs (i.e., AtLEC1, AtABI3, AtFUS3 and AtLEC2) during germination, which is necessary for the transition from seed to seedling development [11, 57, 58]. CsLAV3/5/7 were expressed specifically at late embryogenesis stages of citrus, i.e. CE and (or) E4 stages, suggesting their possible involvement in repression of the SE pathway, for transition to vegetative development.

Another Type II B3 gene CsARF5 (Cs2g09440), the ortholog of AtARF6, showed constitutive expression levels in most of the tissues studied, but expression also peaked in CE (Fig. 5 and Additional file 7). As the cleavage target of miR167, AtARF6 was reported to be required for SE formation, and the arf6 mutant is severely inhibited for SE production in Arabidopsis [33]. In citrus, CsARF5 has also been identified as the target of miR167 in leaf and fruit by degradome sequencing [59]. MiR167 showed low or undetectable expression levels in both EC and NEC (non-embryogenic callus), but accumulated in GE, and reached its peak in subsequently formed CE [40], with a similar expression pattern to CsARF5 (Fig. 5). The accumulation of CsARF5 in CE may suggest its involvement in late embryogenesis, whereas the non-antagonistic expression patterns between miR167 and CsARF5 may result from fine-tuning of miRNA and/or post-transcriptional regulation.

During the SE process, the Type III B3 gene CsARF12 was down-regulated progressively during the differentiation process (E0-E4), but up-regulated in CE (Fig. 5). It has been reported that AtARF16, an ortholog of CSARF12 in Arabidopsis, regulates the expression of AtABI3 in enhancing seed dormancy and ABA-mediated inhibition of seed germination [60]. The elevation of CsABI3 (CsLAV2) and CsARF12 expression levels at the $\mathrm{CE}$ stage might be involved in inhibition of germination at the late embryogenesis stages.

The expression level of two B3 genes (CsARF7 and CsREM9), which were classified as Type IV, increased progressively during the citrus SE process (Fig. 5). AtARF1, which is homologous to CsARF7 (Cs3g01570), binds to auxin response elements and confers auxin responsiveness in development [61]. In citrus, endogenous IAA levels likewise gradually increase during SE and reach a peak in CE [44]. However, the level of endogenous IAA was relatively lower in EC with greater potential for SE, suggesting that auxin may not be a key factor for determination of SE competence [44]. In addition to auxin, a high ratio of ABA to GA was shown to contribute to citrus SE. The accumulation of auxin has previously been reported to modulate the levels of $A B A$ and $\mathrm{GA}$ in regions of future organogenesis [10]. Thus, the association of CsARF7 and the plant hormone auxin, ABA and GA in citrus SE remains to be elucidated. 
The CsREM9 gene belongs to the poorly characterized REM family. Expression and genetic analyses showed that one REM gene $(A t V D D)$ is required for cell differentiation in the female gametophyte and is highly expressed during early stages of seed formation [39]. SE shares morphological, cytological, and molecular similarities with zygotic embryogenesis (ZE) $[44,62]$. The increased expression of CsREM9 during the early stages of SE suggests that CsREM9 may be functional in early embryogenesis as well (Fig. 5).

\section{CSARF19 might be involved in citrus EC initiation}

In citrus, EC can be induced in vitro from the undeveloped ovules/ aborted seeds of the polyembryonic genotypes, but not from the monoembryonic genotypes, which suggests that the regenerative EC might be derived from pluripotent nucellar embryo initiation (NEI) cells localized in the apomictic nucellus tissues [63]. In this study, we identified a candidate B3 TF possibly involved in EC initiation (Fig. 6). ARF19 was identified in mandarin and sweet orange, but not in pummelo or related genera of citrus. In addition, CsARF19 was expressed at a relatively high level in EC compared to other tissues in sweet orange (Fig. $3 \mathrm{~b}$ ). In our previous study, ARF19 was moderately expressed (RPKM values ranged from 15.69 to 34.50) in the ovules of two mandarin cultivars (the monoembryonic 'Nour' Clementine and the polyembryonic 'Huagan No.2' Ponkan) during nucellar embryo initiation, but was expressed at slightly higher levels in the polyembryonic cultivar [64]. However, ARF19 was not expressed $(\mathrm{RPKM}=0)$ in ovules of the monoembryonic 'Huanong red' pummelo, but accumulated in ovules of the polyembryonic 'Cocktail' grapefruit, with RPKM values of 1.34 and 3.58 prior to and during nucellar embryo initiation [64]. Based on the fact that sweet orange and grapefruit were derived from hybridizations between mandarin and pummelo [46, 51, 64], we suggest that CsARF19 originated in mandarin, and was introgressed into the hybrid pool. SE initiation is believed to require an induction signal that causes somatic cells to change identity [65]. Previous studies have shown that two ARF genes (AtARF7 and AtARF19) directly or indirectly target four auxin-responsive LBD (LATERAL ORGAN BOUNDARIES DOMAIN) genes to regulate callus formation in Arabidopsis regeneration [66]. Callus-induction medium containing a high concentration of 2,4-dicholorophenoxy acetic acid (2,4-D) has also been shown to promote callus induction in citrus $[67,68]$. However, the link between auxin signaling and citrus callus initiation has not yet been established. Our analysis implies that CsARF19 is derived from mandarin, a basic species of citrus, and may be involved in callus initiation process from the nucellus tissues of polyembryonic citrus cultivars.
Overall, the present study indicates that some given members of the B3 superfamily may be involved in citrus $\mathrm{SE}$, especially for late $\mathrm{SE}$ stages. One $\mathrm{B} 3$ gene, CsARF19, was indicated to be associated with nucellarderived callus initiation of polyembryonic citrus cultivars. Although phylogenetic and expression analysis provided clues to the roles of the citrus B3 superfamily in $\mathrm{SE}$, further molecular and biochemical studies are required to investigate whether multiple members of B3 superfamily form a coordinated regulation network to control SE, and whether the expression of some key B3 genes could establish a cellular environment favorable to callus initiation or SE competence enhancement.

\section{Methods}

\section{Plant materials}

Non-embryogenic callus (NEC) and embryogenic callus (EC) of 'Valencia' sweet orange were induced, cultured and preserved as described previously [69]. The plant material was identified by Dr. Zheng Liu, an associate researcher of Fruit and Tea Research Institute, Hubei Academy of Agricultural Sciences. The voucher specimens were deposited at Key Laboratory of Horticultural Plant Biology (Ministry of Education), Huazhong Agricultural University. In brief, NEC was recently induced from epicotyl segments, whereas EC was induced from the aborted seeds and preserved in tissue culture for years. The seeds were harvested from 'Valencia' sweet orange trees in the citrus germplasm respository at Huazhong Agricultural University. EC was transferred to glycerol medium to induce SE. Samples were collected from NEC, EC, E2/4 (EC induced from somatic embryos for 2 or 4 weeks), GE and CE. All samples were immediately frozen in liquid nitrogen and stored at $-80^{\circ} \mathrm{C}$ for further analysis.

\section{Genome-wide identification of B3 superfamily genes}

A HMM (Hidden Markov Model) profile of the B3 DNA binding domain (PF02362) was downloaded from the Pfam database (http://pfam.xfam.org/family/PF02362), and exploited for the comprehensive identification of sweet orange and pummelo B3 superfamily genes from the Citrus sinensis Annotation Project (http://citrus.hzau.edu.cn/orange/download/index.php) using HMMER program (version 3.1b2) with a threshold of e-value $<0.01$ [46, 47]. Using the same criterion, B3 family sequences were obtained from Arabidopsis thaliana (ftp://ftp.ensemblgenomes.org/pub/ plants/release-38/fasta/arabidopsis_thaliana/) and Oryza sativa databases (ftp://ftp.ensemblgenomes.org/pub/plants/ release-38/fasta/oryza_sativa/). The conserved domains of all putative candidates were confirmed using InterProScan software package (version 5.25-64.0). Finally, a self-blast of protein sequences was performed to remove redundancy. Alternative splice variants were not considered. Any two 
protein sequences which showed a perfect match were deemed to be redundant gene pairs and the shorter sequence was removed from the potential B3 genes list. Seven draft genomes of citrus species (http://citrus.hzau.edu.cn/orange/download/index.php), including Mangshan mandarin, Clementine mandarin, Atalantia, Ichang papeda, kumquat, trifoliate orange (unpublished data) and citron [47-50], were used to search for orthologs of CsARF19.

\section{Analysis of chromosomal locations, synteny relationships,} protein properties, gene structure and conserved motifs

The physical locations of citrus B3 genes were obtained from the database of sweet orange and pummelo genomes (http://citrus.hzau.edu.cn/orange/download/ index.php). MapChart software (https://www.wur.nl/en/ show/Mapchart.htm) was applied to visualize the distribution of the B3 genes on the citrus chromosomes. To detect gene duplication events, the Multiple Collinearity Scan toolkit (MCScan X) was applied [70]. Dual Synteny Plotter software (https://github.com/CJ-Chen/TBtools) was adopted to exhibit the synteny relationship of the orthologous B3 genes between citrus and Arabidopsis as well as between citrus and rice. The Ks and Ka were calculated using KaKs_Calculator 2.0 [71].

The theoretical isoelectric points and molecular weights of the citrus B3 proteins were predicted by the compute $\mathrm{pI} / \mathrm{Mw}$ tool in the ExPASY server (https://web. expasy.org/compute_pi/). The Gene Structure Display Server (GSDS, http://gsds.cbi.pku.edu.cn/) program was exploited to illustrate exon/intron organization according to cDNA and genomic DNA sequences. The InterProScan program (http://www.ebi.ac.uk/interpro/) was used to characterize the domains and motifs of the citrus B3 superfamily. The predicted three-dimensional structure of the citrus B3 domain was generated using the Swiss-Model server by homology modeling [72]. The B3 domain of CsLAV1 (CsFUS3; gene id: Cs2g14320) was chosen as a template, based on the known structure of AtFUS3 (Protein Data Bank code: 6j9b.2) [45].

\section{Multiple sequence alignments and phylogenetic analysis}

Multiple sequence alignments (MSA) of the B3 domain sequences of citrus B3 proteins were performed using the MAFFT algorithm (version 7) [73]. The robustness of the MSA was assessed by the GUIDANCE2 server (http://guidance.tau.ac.il/ver2/) using 100 bootstrap replicates [74]. The alignment was also presented along with the corresponding secondary structure elements.

To investigate the phylogenetic relationship between citrus and Arabidopsis, neighbor-joining (NJ) trees were constructed by MEGA7 software based on the fulllength of the B3 protein sequences [75]. A bootstrap analysis was performed to estimate the reliability of the tree topology, with 1000 replications. The numbers generated for each clade represent the bootstrap support values expressed as percentages. The same method was adopted to construct the NJ phylogenetic trees for the four families of citrus B3 superfamily.

\section{Expression analysis of CsB3s}

To investigate the expression patterns of all $\mathrm{B} 3$ genes in different citrus tissues, the normalized RPKM values of citrus B3 genes were extracted from the previously published RNA-Seq data of leaf, fruit, callus and flower of sweet orange [46] and that of leaf, fruit, ovule and seed of pummelo [47]. The results were visualized by heat map with transformed $\log _{2}(\mathrm{RPKM}+1)$ values using the 'pheatmap' $\mathrm{R}$ package (https://cran.r-project.org/web/ packages/pheatmap/index.html).

Genes that were highly expressed (RPKM values $>10)$ in EC, or specifically accumulated in EC with lower expression level $(1<$ RPKM values $<10)$ were selected for further analysis using qRT-PCR. Total RNA was extracted using the Trizol reagent from EC and somatic embryos of 'Valencia' sweet orange [69], followed by RNA integrity examination on $1.0 \%$ agarose gels stained with ethidium bromide. First strand cDNA was synthesized using the RevertAid ${ }^{\mathrm{m}}$ First Strand cDNA Synthesis Kit (Fermentas, USA). qRT-PCR was performed as described previously [69]. qRT-PCR primer pairs were designed by Primer Premier 5.0 software (Additional file 9). The specificity of the primers was further confirmed with a melting curve analysis after amplification of each tested genes. Each PCR pattern was verified using four biological replicates. Two reference genes, i.e. CiteIF-1A and CitUBL5, shown to be stably expressed during citrus SE [69], were used as internal controls to normalize the qRT-PCR data. Mixtures without template were employed as the negative control. Data was processed using the $\mathrm{Ct}$ method $\left(2^{-\triangle \triangle C T}\right)$ for relative quantification. Statistical analyses were performed using the IBM SPSS Statistics 19 software as described previously [44].

\section{Supplementary information}

Supplementary information accompanies this paper at https://doi.org/10. 1186/s12864-020-6715-9.

Additional file 1 B3 genes identified in the genomes of sweet orange (Citrus sinensis) and pummelo (C. grandis).

Additional file $2 \mathrm{Ka} / \mathrm{Ks}$ calculation of the duplicated B3 gene pairs in sweet orange (Citrus sinensis) and pummelo (C. grandis).

Additional file $\mathbf{3}$ One-to-one orthologous relationships between sweet orange/pummelo and Arabidopsis as well as those between sweet orange/pummelo and rice.

Additional file 4. Three-dimensional models of the B3 domain from citrus. The Ribbon diagram of the CSLAV1 B3 domain was built using the SWISS-MODEL server. The B3 domain consists of seven $\beta$-strands ( $\beta 1-\beta 7)$ that form an open $\beta$-barrel. Two $\alpha$-helices ( $a 1$ and a2) project above and below the $\beta$-barrel. 
Additional file 5. Multiple-sequence alignments of the B3 domain from $L A V, R A V$ and ARF families of citrus. Red circles indicate identical amino acid residues. Color-coded GUIDANCE2 scores are presented for the citrus B3 domain sequences. Confidently aligned residues are colored in shades of magenta, whereas uncertain residues are colored in shades of blue. GUIDANCE2 scores which represent the degree of confidently aligned residues (1 corresponds to 100\% certainty) are plotted below the alignment.

Additional file 6. Multiple-sequence alignments of the B3 domain from the REM family of citrus. Red circles indicate conserved amino acid residues. Color-coded GUIDANCE2 scores are presented for the citrus B3 domain sequences. Confidently aligned residues are colored in shades of magenta, whereas uncertain residues are colored in shades of blue. GUIDANCE2 scores which represent the degree of confidently aligned residues (1 corresponds to 100\% certainty) are plotted below the alignment.

Additional file 7 Phylogenetic tree of the B3 proteins from citrus and Arabidopsis based on the neighbor-joining method using MEGA7 software. The reliability of the predicted tree was tested by bootstrapping with 1000 replicates. The percentage of neighbor-joining bootstrap replications (> 40\%) is shown above each node.

Additional file 8 Lists of B3 genes identified in Arabidopsis and rice. Additional file 9. List of GRT-PCR primers used in this study.

\section{Abbreviations}

SE: Somatic embryogenesis; EC: Embryogenic callus;" TFs: Transcription factors; RPKM: Reads per kilobase per million mapped reads; Ka/Ks: Nonsynonymous/synonymous substitution ratio; NEC: Non-embryogenic callus; E0/2/4: EC induced for somatic embryos for 0, 2, 4 weeks; GE: Globular embryos; CE: Cotyledon embryos; WGD: Whole-genome duplication; NJ: Neighbor-joining; MSA: Multiple sequence alignments.

\section{Acknowledgements}

Not applicable.

\section{Authors' contributions}

ZL, XMW and WWG conceived and designed the research. ZL and XXG performed the experiments. ZL analyzed the data, and QX participated in data analysis. ZL and XMW wrote the paper. RGA and WWG revised the paper. All authors have read and approved the manuscript for publication.

\section{Funding}

This work was financially supported by the Ministry of Science and Technology of China (no. 2018YFD1000106), the National Natural Science Foundation of China [nos. 31701906, 31872051, 31872084], and the Fundamental Research Funds for Central Universities (nos. 2662018PY007, 2662018PY013). The funders had no role in study design, data collection and analysis, decision to publish, or preparation of the manuscript.

\section{Availability of data and materials}

The sequences information analyzed during the current study are available in the Citrus sinensis Annotation Project (http://citrus.hzau.edu.cn/orange/ download/index.php), Arabidopsis thaliana databases (ftp://ftp. ensemblgenomes.org/pub/plants/release-38/fasta/arabidopsis_thaliana/) and Oryza sativa databases (ftp://ftp.ensemblgenomes.org/pub/plants/release-38/ fasta/oryza_sativa/). The public RNA-seq data was from the Citrus sinensis Annotation Project and NCBI's Sequence Read Archive (SRA) database (the accession number PRJNA339650). All data analyzed during this study are included in this published article and its supplementary information files.

\section{Ethics approval and consent to participate}

Not applicable.

\section{Consent for publication}

Not applicable.

\section{Competing interests}

The authors declare that they have no competing interests.

\section{Author details}

${ }^{1}$ Fruit and Tea Research Institute, Hubei Academy of Agricultural Sciences, Wuhan 430064, China. ${ }^{2}$ Key Laboratory of Horticultural Plant Biology (Ministry of Education), Huazhong Agricultural University, Wuhan 430070, China. ${ }^{3}$ Center of Applied Biotechnology, Wuhan University of Bioengineering, Wuhan 430415, China. ${ }^{4}$ The New Zealand Institute for Plant \& Food Research Limited (PFR), Private Bag 92169, Auckland 1142, New Zealand.

Received: 19 November 2019 Accepted: 3 April 2020

Published online: 16 April 2020

\section{References}

1. Swaminathan K, Peterson K, Jack T. The plant B3 superfamily. Trends Plant Sci. 2008;13:647-55.

2. Yamasaki K, Kigawa T, Seki M, Shinozaki K, Yokoyama S. DNA-binding domains of plant-specific transcription factors: structure, function, and evolution. Trends Plant Sci. 2013:18:267-76.

3. Suzuki M, Kao CY, McCarty DR. The conserved B3 domain of VIVIPAROUS1 has a cooperative DNA binding activity. Plant Cell. 1997;9:799-807.

4. Romanel EAC, Schrago CG, Counago RM, Russo CAM, Alves-Ferreira M. Evolution of the B3 DNA binding superfamily: new insights into REM family gene diversification. PLoS One. 2009;4(6):e5791.

5. Peng FY, Weselake RJ. Genome-wide identification and analysis of the B3 superfamily of transcription factors in Brassicaceae and major crop plants. Theor Appl Genet. 2013;126:1305-19.

6. Xia F, Sun T, Yang S, Wang $X$, Chao J, Li X, et al. Insight into the B3Transcription Factor Superfamily and Expression Profiling of B3 Genes in Axillary Buds after Topping in Tobacco (Nicotiana tabacum L.). Gene. 2019; 10:164.

7. Ahmad B, Zhang S, Yao J, Rahman MU, Hanif M, Zhu Y, et al. Genomic organization of the B3-domain transcription factor family in Grapevine (Vitis vinifera L.) and expression during seed development in seedless and seeded sultivars. Int J Mol Sci. 2019;20:4553.

8. Nambara E, Hayama R, Tsuchiya Y, Nishimura M, Kawaide H, Kamiya Y, et al. The role of $A B / 3$ and FUS3 loci in Arabidopsis thaliana on phase transition from late embryo development to germination. Dev Biol. 2000;220:412-23.

9. Stone SL, Kwong LW, Yee KM, Pelletier J, Lepiniec L, Fischer RL, et al. LEAFY COTYLEDON2 encodes a B3 domain transcription factor that induces embryo development. Proc Natl Acad Sci. 2001;98:11806-11.

10. Gazzarrini S, Tsuchiya Y, Lumba S, Okamoto M, McCourt P. The transcription factor FUSCA3 controls developmental timing in Arabidopsis through the hormones gibberellin and abscisic acid. Dev Cell. 2004;7:373-85.

11. Suzuki M, Wang HHY, MCCarty DR. Repression of the LEAFY COTYLEDON 1/ $B 3$ regulatory network in plant embryo development by VPI/ABSCISIC ACID INSENSITIVE 3-LIKE B3 genes. Plant Physiol. 2007;143:902-11.

12. Veerappan $V$, Wang J, Kang M, Lee J, Tang Y, Jha AK, et al. A novel HSI2 mutation in Arabidopsis affects the PHD-like domain and leads to derepression of seed-specific gene expression. Planta. 2012;236:1-17.

13. Wojcikowska B, Jaskota K, Gasiorek P, Meus M, Nowak K, Gaj MD. LEAFY COTYLEDON2 (LEC2) promotes embryogenic induction in somatic tissues of Arabidopsis, via YUCCA-mediated auxin biosynthesis. Planta. 2013;238:425-40.

14. Suzuki M, McCarty DR. Functional symmetry of the B3 network controlling seed development. Curr Opin Plant Biol. 2008;11:548-53.

15. Carbonero P, Iglesias-Fernandez R, Vicente-Carbajosa J. The AFL subfamily of B3 transcription factors: evolution and function in angiosperm seeds. J Exp Bot. 2016;203:817-29.

16. Tsukagoshi H, Morikami A, Nakamura K. Two B3 domain transcriptional repressors prevent sugar-inducible expression of seed maturation genes in Arabidopsis seedlings. Proc Natl Acad Sci. 2007:104:2543-7.

17. Monke G, Altschmied L, Tewes A, Reidt W, Mock HP, Baumlein H, et al. Seed-specific transcription factors ABI3 and FUS3: molecular interaction with DNA. Planta. 2004;219:158-66.

18. Jia H, Suzuki M, McCarty RM. Regulation of the seed to seedling developmental phase transition by the LAFL and VAL transcription factor networks. Wires Dev Biol. 2013;3:135-45.

19. Matias-Hernandez L, Aguilar-Jaramillo AE, Marin-Gonzalez E, Suarez-Lopez P, Pelaz S. RAV genes: regulation of floral induction and beyond. Ann Bot. 2014;114:1459-70.

20. Hu YX, Wang YH, Liu XF, Li JY. Arabidopsis RAV1 is down-regulated by brassinosteroid and may act as a negative regulator during plant development. Cell Res. 2004;14:8-15. 
21. Woo HR, Kim JH, Kim J, Kim J, Lee U, Song IJ, et al. The RAV1 transcription factor positively regulates leaf senescence in Arabidopsis. J Exp Bot. 2010;61:3947-57.

22. Li CW, Su RC, Cheng CP, Sanjaya YSJ, Hsieh TH, et al. Tomato RAV transcription factor is a pivotal modulator involved in the AP2/EREBPmediated defense pathway. Plant Physiol. 2011;156:213-27.

23. Fu M, Kang HK, Son SH, Kim SK, Nam KH. A subset of Arabidopsis RAV transcription factors modulates drought and salt stress responses independent of ABA. Plant Cell Physiol. 2014;55:1892-904.

24. Lu Q, Zhao L, Li D, Hao D, Zhan Y, Li W. A GmRAV ortholog is involved in photoperiod and sucrose control of flowering time in soybean. PLoS One. 2014;9:e89145.

25. Lee BH, Kwon SH, Lee SJ, Park SK, Song JT, Lee S, et al. The Arabidopsis thaliana NGATHA transcription factors negatively regulate cell proliferation of lateral organs. Plant Mol Biol. 2015;89:529-38.

26. Zhao SP, Xu ZS, Zheng WJ, Zhao W, Wang YX, Yu TF, et al. Genome-wide analysis of the RAV family in soybean and functional identification of GmRAV-03 involvement in salt and drought stresses and exogenous ABA treatment. Front Plant Sci. 2017:8:905.

27. Guilfoyle TJ, Hagen G. Getting a grasp on domain III/IV responsible for Auxin response factor-IAA protein interactions. Plant Sci. 2012;190:82-8.

28. Boer DR, Freire-Rios A, van den Berg WAM, Saaki T, Manfield IW, Kepinski S, et al. Structural basis for DNA binding specificity by the Auxin-dependent ARF transcription factors. Cell. 2014;156:577-89.

29. Kumar R, Tyagi AK, Sharma AK. Genome-wide analysis of auxin response factor (ARF) gene family from tomato and analysis of their role in flower and fruit development. Mol Genet Genomics. 2011;285:245-60.

30. Breitel DA, Chappell-Maor L, Meir S, Panizel I, Puig CP, Hao Y, et al. AXUIN RESPONSE FACTOR2 intersects hormonal signals in the regulation of tomato fruit repening. PLoS Genet. 2016;12(3):e1005903.

31. Ge J, Li B, Shen D, Xie J, Long J, Dong H. Tobacco TTG2 regulates vegetative growth and seed production via the predominant role of ARF8 in cooperation with ARF17 and ARF19. BMC Plant Biol. 2016;16:126.

32. Krogan NT, Marcos D, Weiner Al, Berleth T. The auxin response factor MONOPTEROS controls meristem function and organogenesis in both the shoot and root through the direct regulation of PIN genes. New Phytol. 2016;212:42-50.

33. Su YH, Liu YB, Zhou C, Li XM, Zhang XS. The microRNA167 controls somatic embryogenesis in Arabidopsis through regulating its target genes ARF6 and ARF8. Plant Cell Tissue Organ Cult. 2016;124:405-17.

34. Ren Z, Liu R, Gu W, Dong X. The Solanum lycopersicum auxin response factor SIARF2 participates in regulating lateral root formation and flower organ senescence. Plant Sci. 2017;256:103-11.

35. Wojcikowska B, Gaj MD. Expression profiling of AUXIN RESPONSE FACTOR genes during somatic embryogenesis induction in Arabidopsis. Plant Cell Rep. 2017;36:843-58.

36. Mantegazza O, Gregis V, Mendes MA, Morandini P, Alves-Ferreria M, Patreze CM, et al. Analysis of the arabidopsis REM gene family predicts functions during flower development. Ann Bot. 2014;114:1507-15.

37. Franco-Zorrilla JM, Cubas P, Jarillo JA, Fernandez-Calvin B, Salinas J, Martinez-Zapater JM. AtREM1, a member of a new family of B3 domaincontaining genes, is preferentially expressed in reproductive meristems. Plant Physiol. 2002;128:418-27.

38. Levy YY, Mesnage S, Mylne JS, Gendall AR, Dean C. Multiple roles of Arabidopsis VRN1 in vernalization and flowering time control. Science. 2002; 297:243-6.

39. Matias-Hernandez L, Battaglia R, Galbiati F, Rubes M, Eichenberger C, Grossniklaus $U$, et al. VERDAND1 is a direct target of the MADS domain ovule identity complex and affects embryo sac differentiation in Arabidopsis. Plant Cell. 2010;22:1702-15.

40. Wu XM, Liu MY, Ge XX, Xu Q, Guo WW. Stage and tissue-specific modulation of ten conserved miRNAs and their targets during somatic embryogenesis of Valencia sweet organe. Planta. 2011;233:495-505.

41. Ge XX, Chai LJ, Liu Z, Wu XM, Deng XX, Guo WW. Transcriptional profiling of genes involved in embryogenic, non-embryogenic calluses and somatic embryogenesis of Valencia sweet orange by SSH-based microarray. Planta. 2012;236:1107-24.

42. Pan ZY, Guan R, Zhu SP, Deng XX. Proteomic analysis of somatic embryogenesis in Valencia sweet orange (Citrus sinensis Osbeck). Plant Cell Rep. 2009;28:281-9.

43. Wu XM, Kou SJ, Liu YL, Fang YN, Xu Q, Guo WW. Genome-wide analysis of small RNAs in non-embryogenic and embryogenic tissues of citrus:
microRNA and siRNA mediated transcript cleavage involved in somatic embryogenesis. Plant Biotechnol J. 2015;13:383-94.

44. Liu Z, Ge XX, Qiu WM, Long JM, Jia HH, Yang W, et al. Overexpression of the CSFUS3 gene encoding a B3 transcription factor promotes soamtic embryogenesis in Citrus. Plant Sci. 2018;227:121-31.

45. Tao Z, Hu H, Luo X, Jia B, Du J, He Y. Embryonic resetting of the parental vernalized state by two B3 domain transcription factors in Arabidopsis. Nat Plants. 2019;5:424-35.

46. Xu Q, Chen LL, Ruan XA, Chen DJ, Zhu AD, Chen $C L$, et al. The draft genome of sweet orange (Citrus sinensis). Nat Genet. 2013;45:59-66.

47. Wang X, Xu Y, Zhang S, Cao L, Huang Y, Cheng J, et al. Genomic analyses of primitive, wild and cultivated citrus provide insights into asexual reproduction. Nat Genet. 2017;49:765-72.

48. Wang L, He F, Huang Y, He J, Yang S, Zeng J, et al. Genome of wild mandarin and domestication history of mandarin. Mol Plant. 2018;11:102437.

49. Zhu C, Zheng X, Huang Y, Ye J, Chen P, Zhang C, et al. Genome sequencing and CRISPR/Cas 9 gene editing of an early flowering mini-Citrus (Fortunella hindsii). Plant Biotechnol J. 2019;17:2199-210.

50. Wu GA, Prochnik S, Jenkins J, Salse J, Hellsten U, Murat F, et al. Sequencing of diverse mandarin, pummelo and orange genomes reveals complex history of admixture during citrus domestication. Nat Biotechnol. 2014;32: 656-62.

51. Wu GA, Terol J, Ibanez V, López-García A, Pérez-Román E, Borredá C, et al. Genomics of the origin and evolution of Citrus. Nature. 2018;554:311-6.

52. The Arabidopsis Genome Initiative. Analysis of the genome sequence of the flowering plant Arabidopsis thaliana. Nature. 2000;408:796-815.

53. Yu J, Hu S, Wang J, Wong GKS, Li S, Liu B, et al. A draft sequence of the rice genome (Oryza sativa L. ssp. indica). Science. 2002;296:79-92.

54. Ckurshumova W, Smirnova T, Marcos D, Zayed Y, Berleth T. Irrepressible MONOPTEROS/ARF5 promotes de novo shoot formation. New Phytol. 2014; 204:556-66.

55. Parcy F, Valon C, Raynal M, Gaubier-Comella P, Delseny M, Giraudat J. Regulation of gene expression programs during Arabidopsis seed development: roles of the $A B / 3$ locus and of endogenous abscisic acid. Plant Cell. 1994:6:1567-82.

56. Braybrook SA, Stone SL, Park S, Bui AQ, Le BH, Fischer RL, et al. Genes directly regulated by LEAFY COTYLEDON2 provide insight into the control of embryo maturation and somatic embryogenesis. Proc Natl Acad Sci. 2006;103:3468-73.

57. Schneider A, Aghamirzaie D, Elmarakeby H, Poudel AN, Koo AJ, Heath LS, et al. Potential targets of VIVIPAROUS1/ABI3-LIKE1 (VAL1) repression in developing Arabidopsis thaliana embryos. Plant J. 2016;85:305-19.

58. Jia H, McCarty DR, Suzuki M. Distinct roles of LAFL network genes in promoting the embryonic seedling fate in the absence of VAL repression. Plant Physiol. 2013;163:1293-305.

59. Liu Y, Wang L, Chen D, Wu X, Huang D, Chen L, et al. Genome-wide comparison of microRNAs and their targeted transcripts among leaf, flower and fruit of sweet orange. BMC Genomics. 2014;15:695.

60. Liu X, Zhang H, Zhao Y, Feng Z, Li Q, Yang HQ, et al. Auxin controls seed dormancy through stimulation of abscisic acid signaling by inducing ARFmediated ABI3 activation in Arabidopsis. Proc Natl Acad Sci. 2013;110:15485-90.

61. Ulmasov T, Gretchen H, Guilfoyle TJ. ARF1, a transcription factor that binds to auxin response elements. Science. 1997;276:1865-8.

62. Rupps A, Raschke J, Rümmler M, Linke B, Zoglauer K. Identification of putative homologs of Larix decidua to BABYBOOM (BBM), LEAFY COTYLEDON1 (LEC1), WUSCHEL-related HOMEOBOX2 (WOX2) and SOMATIC EMBRYOGENESIS RECEPTOR-like KINASE (SERK) during somatic embryogenesis Planta. 2016:243:473-88.

63. Miah MN, Islam S, Hadiuzzaman S. Regeneration of plantlets through somatic embryogenesis from nucellus tissue of Citrus macroptera Mont. Var. anammensis ('sat Kara'). Plant Tissue Cult. 2002;12:167-72.

64. Long JM, Liu Z, Wu XM, Fang YN, Jia HH, Xie ZZ, et al. Genome-scale mRNA and small RNA transcriptomic insights into initiation of citrus apomixis. J Exp Bot. 2016;67:5743-56.

65. Uddenberg D, Abrahamsson M, von Arnold S. Overexpression of PaHAP3A stimulates differentiation of ectopic embryos from maturing somatic embryos of Norway spruce. Tree Genet Genome. 2016;12:18.

66. Fan $M, X u C$, Xu K, Hu Y. LATERAL ORGAN BOUNDARIES DOMAIN transcription factors direct callus formation in Arabidopsis regeneration. Cell Res. 2012;22:1169-80. 
67. Savita SB, Virk GS, Nagpal AK. An efficient plant regeneration protocol from callus cultures of Citrus jambhiri Lush. Physiol Mol Biol Pla. 2011;17:161-9.

68. Hussain M, Raja NI, Iqbal M, Iftikhar A, Sadaf HM, Sabir S, et al. Plantlets regeneration via somatic embryogenesis from the nucellus tissues of Kinnow mandarin (Citrus reticulata L.). American J Plant Sci. 2016;7:798-805.

69. Liu Z, Ge XX, Wu XM, Kou SJ, Chai LJ, Guo WW. Selection and validation of suitable reference genes for mRNA qRT-PCR analysis using somatic embryogenic cultures, floral and vegetative tissues in citrus. Plant Cell Tiss Org. 2013;113:469-81.

70. Wang Y, Tang H, DeBarry JD, Tan X, Li J, Wang X, et al. MCScan X: a toolkit for detection and evolutionary analysis of gene synteny and collinearity. Nucleic Acids Res. 2012;40:e49.

71. Wang D, Zhang Y, Zhang Z, Zhu J, Yu J. KaKs_Calculator 2.0: a toolkit incorporating gamma-series methods and sliding window strategies. Genomics Proteomics Bioinformatics. 2010;8:77-80.

72. Waterhouse A, Bertoni M, Bienert S, Studer G, Tauriello G, Gumienny R, et al. SWISS-MODEL: homology modelling of protein structures and complexes. Nucleic Acids Res. 2018;46:W296-303.

73. Katoh K, Standley DM. MAFFT multiple sequence alignment software version 7: improvements in performance and usability. Mol Biol Evol. 2013: 30:772-80.

74. Sela I, Ashkenazy H, Katoh K, Pupko T. GUIDANCE2: accurate detection of unreliable alignment regions accounting for the uncertainty of multiple parameters. Nucleic Acids Res. 2015;43:W7-W14.

75. Kumar S, Stecher G, Tamura K. MEGA7: molecular evolutionary genetics analysis version 7.0 for bigger datasets. Mol Biol Evol. 2016;33:1870-4.

\section{Publisher's Note}

Springer Nature remains neutral with regard to jurisdictional claims in published maps and institutional affiliations.

Ready to submit your research? Choose BMC and benefit from:

- fast, convenient online submission

- thorough peer review by experienced researchers in your field

- rapid publication on acceptance

- support for research data, including large and complex data types

- gold Open Access which fosters wider collaboration and increased citations

- maximum visibility for your research: over $100 \mathrm{M}$ website views per year

At $\mathrm{BMC}$, research is always in progress.

Learn more biomedcentral.com/submissions 